EPJ Web of Conferences 80,00019 (2014)

DOI: $10.1051 /$ epj conf/ 20148000019

(C) Owned by the authors, published by EDP Sciences, 2014

\title{
Hadronization systematics and top mass reconstruction
}

\author{
Gennaro Corcella ${ }^{1, a}$ \\ ${ }^{1}$ INFN, Laboratori Nazionali di Frascati, Via E. Fermi 40, I-00044, Frascati (RM), Italy
}

\begin{abstract}
I discuss a few issues related to the systematic error on the top mass measurement at hadron colliders, due to hadronization effects. Special care is taken about the impact of bottom-quark fragmentation in top decays, especially on the reconstruction relying on final states with leptons and $J / \psi$ in the dilepton channel. I also debate the relation between the measured mass and its theoretical definition, and report on work in progress, based on the Monte Carlo simulation of fictitious top-flavoured hadrons, which may shed light on this issue and on the hadronization systematics.
\end{abstract}

\section{Introduction}

Heavy-flavour, and in particular top-quark phenomenology is nowadays one of the most lively fields of investigation, in both theoretical and experimental particle physics. At hadron colliders, top-quark pairs are mainly produced through strong interactions and decay via $t \rightarrow b W$ before hadronizing; the final states are then classified as dileptons, leptons+jets or all-jets, according to the decay mode of the $W$ boson. The top-quark mass is then measured by reconstructing its decay products.

In fact, the top-quark mass $m_{t}$ is a fundamental parameter of the Standard Model, as it has been employed, together with the $W$ mass, even before the discovery of the Higgs boson [1], in the electroweak precision tests constraining the Higgs mass [2]. Furthermore, using its current world average, i.e. $m_{t}=173.34 \pm 0.27$ (stat) \pm 0.71 (syst) GeV [3], and the Higgs mass results, one finds that the Standard Model vacuum lies at the border between stability and metastability regions [4]. For the above reasons, it is clear that it is necessary to have under control all possible sources of uncertainties on the top mass determination, such as the theory systematics, and that it would be desirable to measure $m_{t}$ with the highest possible precision.

In this paper I investigate the uncertainties on the measurement of the top mass, paying special attention to the hadronization corrections, such as the modelling of bottom-quark fragmentation in top decays. In fact, $b$-quark fragmentation enters in the theoretical uncertainty on the top mass reconstruction, as it contributes to the Monte Carlo systematics. The world average determination, based on standard reconstruction methods, such as the template, ideogram and matrix-element techniques. quotes an overall Monte Carlo uncertainty of $380 \mathrm{MeV}$, whereas the systematic error due to $b$-tagging and $b$-jet energy scale, also depending on bottom fragmentation in top decays, are about 250 and $110 \mathrm{MeV}$, respectively. An even larger impact of the treatment of the hadronization mechanism is found in the method relying on final states where both $W$ 's decay leptonically $(W \rightarrow \ell v)$ and the $B$

a e-mail: gennaro.corcella@lnf.infn.it 
hadron into a $J / \psi$ [5]. Although the decay $B \rightarrow J / \psi X$ is a rare one, the events where the $J / \psi$ decays via $J / \psi \rightarrow \mu^{+} \mu^{-}$give a fully leptonic final state, in such a way that, after setting suitable cuts on lepton rapidities and transverse momenta, a measurement of the peak value of the three-lepton invariant mass $m_{3 \ell}$ or of the $J / \psi \ell$ one, i.e. $m_{J / \psi \ell}$, allows a reliable fit of $m_{t}$. The total systematic error is $\Delta m_{t} \simeq 1.47 \mathrm{GeV}$ [6], with $1.37 \mathrm{GeV}$ due to the theoretical uncertainties; the contribution of $b$-quark fragmentation to the systematics, estimated by running the PYTHIA event generator [7], is about $0.7 \mathrm{GeV}$. Refs. [8] and [9] further investigated this issue and compared PYTHIA with HERWIG [10], after fitting their hadronization models to LEP and SLD data: it was then stated that the actual uncertainty can be even larger than what estimated in $[5,6]$ using only one event generator.

In this paper I address some topics which are relevant for the sake of a reliable estimate of the theory uncertainty on the top mass extraction. In Section 2, I reconsider the findings of [8, 9] and compare the Monte Carlo predictions with the NLO calculation [11] as well. In Section 3 I briefly discuss the relation between reconstructed mass and theoretical definitions, such as the pole mass, and present some ideas to address this issue, based on the Monte Carlo simulation of fictitious top-hadron states. Section 4 contains some concluding remarks.

\section{Bottom-fragmentation in top decays and impact on the theory systematics}

Bottom-quark fragmentation in top decays has been thoroughly investigated through the years, according to fixed-order and resummed calculations, as well as Monte Carlo event generators. The total top-quark width has been calculated up to next-to-next-to-leading order (NNLO) accuracy in [12], whereas the $b$-quark energy spectrum has been obtained by means of soft/collinear resummed calculations, based on the perturbative fragmentation approach [13], to next-to-leading-logarithmic (NLL) accuracy $[14,15]$ and matched to the exact NLO result. Hadronization effects are included by convoluting the resummed $b$-quark distribution with a non-perturbative fragmentation function, such as the Kartvelishvili or Peterson models. An alternative, and perhaps theoretically better approach, first advocated in [16] and later used even in [15], consists of working in Mellin moment space, with a $N$-space non-perturbative fragmentation function, without making any assumption on its functional form in $x$-space.

Ref. [11] computed the invariant mass distribution of a charged lepton, coming from $W$ decay, and a $B$ meson at NLO, using the fits in [14] to model non-perturbative corrections. NNLO distributions in top decays, with massless $b$ quarks and without accounting for hadronization effects, were presented in $[17,18]$. While all the above computations have been undertaken in the narrow-width approximation with on-shell top quarks, Ref. [19] took into account interference effects between top production and decay at NLO and accounted for the resummation of some enhanced contributions by using a dynamical renormalization scale, depending on the top-quark transverse momentum. Of course, it would be very interesting to match the parton-level calculations [17-19] with suitable hadronization models.

As far as Monte Carlo generators, such as HERWIG or PYTHIA, are concerned, they include radiative effects by means of parton showers, equivalent to a resummation of leading soft/collinear logarithms (LLs), with the inclusion of some NLLs [20]. The transitions of partons to hadrons is then modelled via the string [21] (PYTHIA) and cluster (HERWIG) [22] models. All non-perturbative models depend on a few free parameters which are be tuned to experimental data; although using the LHC data would be of course desirable, at the moment the best fits employ $e^{+} e^{-} \rightarrow b \bar{b}$ data from LEP and SLD experiments. The best-fit parametrizations can then be used to predict $B$-hadron production in top decays at the LHC, paying attention to the consistency of the procedure, i.e. tunings relying 
on resummed computations or parton showers for $e^{+} e^{-}$annihilation must be used only if $b$-quark ( $B$-hadron) production in top decay is treated in the same manner. When using a formalism entirely developed in moment space, one can fit directly the moments of the non-perturbative fragmentation function and use such moments for other processes, like top decays. LEP and SLD collaborations did tune hadronization models to the $B$-hadron energy spectrum at the $Z$ pole, but every fit yielded different numbers, so that one could not make any final statement on a parametrization which could eventually be implemented into a Monte Carlo code.

Ref. [8] presented an improved tuning of bottom fragmentation, since OPAL [23], ALEPH [24] and SLD [25] data were taken into account altogether, as if they came from the same experimental sample. The finding is that the default versions of PYTHIA 6 and HERWIG 6 were unable to describe $B$-hadron data $\left(\chi^{2} /\right.$ dof $\sim O(10)$ for both codes), but one had to retune the cluster and string models. After the tuning, PYTHIA managed to give a good fit of the data $\left(\chi^{2} /\right.$ dof $\left.\simeq 1\right)$, while HERWIG, although much improved with respect to the default parametrization, still exhibited meaningful discrepancies $\left(\chi^{2} /\right.$ dof $\left.\simeq 3.5\right)$. The drawback of this analysis lies in the fact that only one observable, namely the $B$-hadron energy fraction in the $Z$ rest frame $x_{B}$, was tuned and therefore the agreement with other measurements, e.g. involving light hadrons, might have been spoiled. As for PYTHIA, the universality of the $a, b$ and $r$ parameters of the Lund model [21] is clearly not respected in the parametrization proposed in [8]. The tuning of [8] can thus be improved by choosing values of $a, b$ and $r$ which respect the universality of the Lund model and are capable of reproducing both heavyand light-flavoured hadron data [26]. Also, tuning CLSMR(1), the gaussian smearing for light-hadron production with respect to the direction of the parent quark, was found not to be essential when dealing with top and bottom quarks [26]. Though with these limitations, the fits in [8] were nonetheless used in the jet-energy measurement by the ATLAS Collaboration [27].

The tuning in [8] was also employed in [9], where, extending previous analyses undertaken in $[28,29]$, HERWIG and PYTHIA were used to study the $B$-lepton invariant-mass distribution for $t \bar{t}$ events at the LHC, in the dilepton channel, and possibly extract $m_{t}$ from the measurement of the Mellin moments of $m_{B \ell}$. The $m_{B \ell}$ spectrum is in fact quite interesting, since it is Lorentz-invariant, thus roughly independent of initial-state radiation and $t \bar{t}$ production mechanism, and can be used to obtain, after a convolution with the $B \rightarrow J / \psi X$ one, the $m_{J / \psi \ell}$ invariant mass distribution and then obtain the top mass as explained in $[5,6]$. Eq. (1) presents the linear fits which parametrize the average value $\left\langle m_{B \ell}\right\rangle$ in terms of $m_{t}$, according to HERWIG (HW), PYTHIA (PY), tuned as in [8], and the NLO calculation of [11], carried out within the perturbative fragmentation formalism, taking the non-perturbative information from [14].

$$
\begin{aligned}
&\left\langle m_{B \ell}\right\rangle_{\mathrm{HW}} \simeq-25.31 \mathrm{GeV}+0.61 m_{t} ; \delta=0.043 \mathrm{GeV} \\
&\left\langle m_{B \ell}\right\rangle_{\mathrm{PY}} \simeq-24.11 \mathrm{GeV}+0.59 m_{t} ; \delta=0.022 \mathrm{GeV} \\
&\left\langle m_{B \ell}\right\rangle_{\mathrm{NLO}} \simeq-26.70 \mathrm{GeV}+0.60 m_{t} ; \delta=0.004 \mathrm{GeV}
\end{aligned}
$$

In (1), $\delta$ is the mean square deviation in the fit and the straight lines are plotted in Fig. 1, in the range $171 \mathrm{GeV}<m_{t}<179 \mathrm{GeV}$. As Fig. 1 exhibits, the three predictions are quite different and can lead to a discrepancy $\Delta m_{t}$ between 1 and $2 \mathrm{GeV}$ if $\left\langle m_{B \ell}\right\rangle$ were to be used to reconstruct $m_{t}$. This difference, beyond the intrinsic differences in the PYTHIA, HERWIG and NLO approaches, is mostly driven by the fact that the fits of HERWIG to the $e^{+} e^{-}$data were not very satistactory. In perspective, using the object-oriented PYTHIA 8 [30] and HERWIG++ [31] codes can be a valuable strategy for a reliable description of bottom-quark fragmentation in the framework of Monte Carlo generators, since both $\mathrm{C}++$ programs manage to reproduce quite successfully the $e^{+} e^{-}$data, with parametrizations which work well even for light hadrons [32]. 


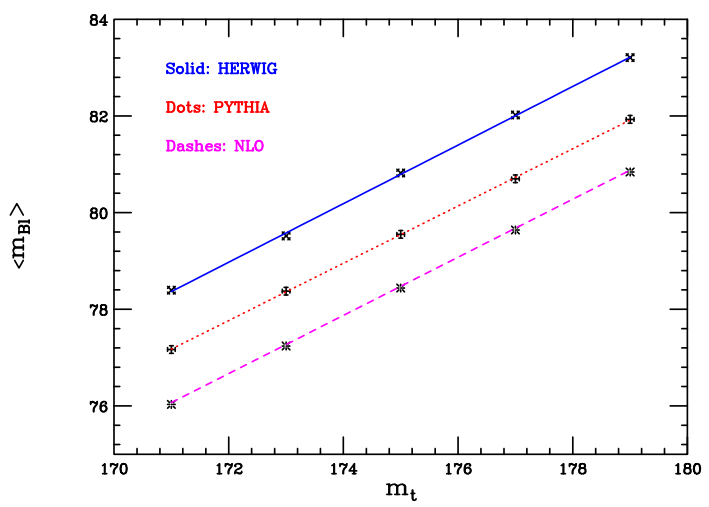

Figure 1. Average value of the invariant mass $m_{B \ell}$ as a function of the top mass, according to HERWIG, PYTHIA and the NLO calculation, tuned to $e^{+} e^{-}$data from LEP and SLD.

\section{Reconstructed mass and theoretical definitions}

The reconstructed top mass, based on the template, ideogram and matrix-element techniques, or even the lately proposed endpoint [33] and $J / \psi$ [6] methods, is obtained by comparing data with predictions yielded by Monte Carlo simulations (see, e.g., Ref. [34] for an update). Since such codes are not exact NLO calculations, the reconstructed mass cannot be precisely identified with any theoretical definition, such as the pole or $\overline{\mathrm{MS}}$ masses. The improved POWHEG [35] and MC@NLO [36] programs, used, e.g., in the analysis [37] to extract the top mass from leptonic observables in the dilepton channel, do yield the total NLO cross section, but still rely on HERWIG or PYTHIA for showers and hadronization. In particular, generators like HERWIG and PYTHIA factorize top production and decay, thus neglecting interference and width effects, which should instead be accounted in a full NLO computation. Therefore, one usually refers to the measured $m_{t}$ as a so-called 'Monte Carlo mass'.

However, the very fact that $m_{t}$ is determined from the kinematics of top-decay products, assuming on-shell top quarks, should lead to the conclusion that it must be close to the pole mass. In fact, analyses carried out in the framework of Soft Collinear Effective Theory for $e^{+} e^{-} \rightarrow t \bar{t}$ annihilation, wherein the Monte Carlo top mass can be associated with the jet mass evaluated at a scale of the order ot the shower cutoff, i.e. about $1 \mathrm{GeV}$, have argued that the reconstructed mass differs from the pole mass by an amount $\sim O\left(\alpha_{S} \Gamma_{t}\right)$ [38], $\Gamma_{t}$ being the top width. Furthermore, the comparison of the measured $t \bar{t}$ cross section [40] with the complete NNLO+NNLL calculation in [39], making use of the pole mass, has proved that the pole mass is indeed compatible with the one determined by means of the standard final-state reconstruction. Nevertheless, the error on $m_{t}$, when extracted from the cross section measurement, is still too large to make this method, albeit very interesting and theoretically consistent, really competitive with respect to the template or matrix-element techniques [40].

In order to tackle the issue of relating Monte Carlo and pole masses, a new investigation, based on the simulation of fictitious top-flavoured hadrons, was recently proposed [26]. In fact, if a quark is confined in a hadron of a given mass, it is well known how it is possible to determine its mass, in both on-shell and $\overline{\mathrm{MS}}$ renormalization schemes, through, e.g., lattice methods, Non Relativistic QCD or Heavy Quark Effective Theories. In fact, one can modify the shower and hadronization models in HERWIG or PYTHIA in such a way that the top quark hadronizes, e.g., in $T^{0}=t \bar{u}$ or $T^{-}=\bar{t} d$ mesons, which then decay according to the spectator model. In such processes, the light quark is 


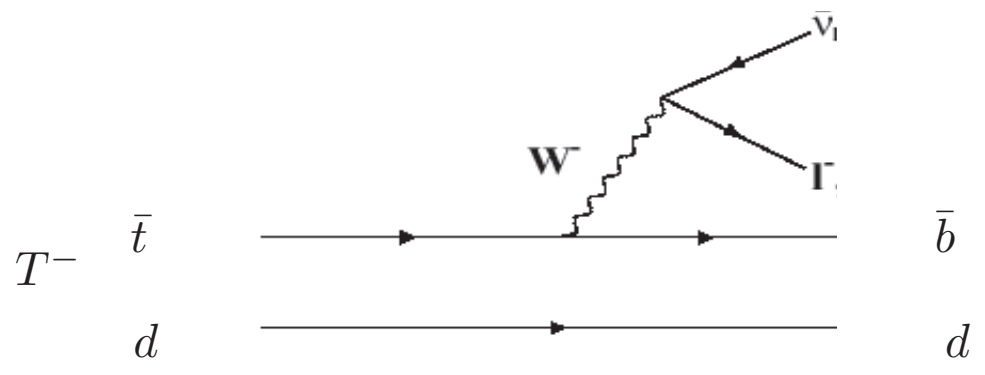

Figure 2. Diagram with a fictitious top-flavoured hadron $T^{-}$decaying according to the spectator model.

the spectator one and the active $t$-quark undergoes a $t \rightarrow b W$ transition, as displayed in Fig. 2 for a $T^{-}$meson; the $b$ and light quark possibly radiate further gluons and finally hadronize. One can then study final-state distributions for this sample of events, such as the $B \ell$ invariant mass discussed above, and reconstruct the top mass from its Mellin moments. If one compares such distributions with those obtained in standard events, i.e. top quarks decaying without hadronizing, one should be able to relate the Monte Carlo mass with the $T$-meson mass and eventually with pole or $\overline{\mathrm{MS}}$ masses. This investigation can therefore be a useful benchmark to address the nature of the Monte Carlo mass and the hadronization systematics. Moreover, the simulation of hadronized top quarks can also give some insight on the impact of colour-reconnection effects. In standard samples, the top quark gets its colour from an initial-state gluon or light quark and gives it to the bottom quark. When hadronizing, it must instead create a colour singlet with the light (spectator) quark, which may yield a different colour flow in the whole event.

Preliminary results of this analysis, already presented in [26] by using the HERWIG event generator, are quite cumbersome. Above all, it was found that, in the hadronized samples, the spectator quark hardly radiates and there is even a non-negligible fraction of events, proportional to the shower Sudakov form factor [20], where the $b$ quark does not emit any gluon either. When this is the case, typically the $b$ quark forms a cluster of small invariant mass, thus capable of decaying just into a $B$ meson plus a soft hadron, e.g. a soft pion. On the contrary, in standard top decays, the bottom quarks can radiate several soft/collinear gluons and give rise to quite energetic clusters. Considering the $B W$ invariant mass, as a toy-observable sensitive to the top-quark mass, its spectrum is in general quite different according to whether the top quark hadronizes or not. Selecting only those events where in both simulations the $b$ quark emits gluons will make the $m_{B W}$ spectra look alike. Table 1 presents the the Mellin moments of $m_{B W}$ for the events with gluon radiation from the $b$ quarks as a function of the Monte Carlo top mass in the range $171 \mathrm{GeV}<m_{t}<179 \mathrm{GeV}$. One can learn that the Mellin moments of $m_{B \ell}$ when the top quark is forced to hadronize before decaying are slightly larger than those in the standard samples, by an amount which runs from $1 \%\left(\left\langle m_{B \ell}\right\rangle\right)$ to $4 \%\left(\left\langle m_{B \ell}^{4}\right\rangle\right)$. Such results, though quite stimulating, are not conclusive and need further investigation: for example, rather than a $B$ meson, one should consider the jet containing the $B$ meson and apply the same jet-clustering algorithms as the experimental analyses. Also, it will be very interesting investigating how much the numbers in Table 1 depend on the shower cutoff and whether it may be possible modifying the decay model, e.g. by means of a Lorentz boost, in such a way that even the spectator quark is allowed to radiate. This is in progress. 
Table 1. First four Mellin moments of the $m_{B W}$ invariant mass distribution yielded by HERWIG with hadronized top quarks. In brackets, the moments for standard $t \bar{t}$ events are quoted.

\begin{tabular}{lllll}
\hline$m_{t}(\mathrm{GeV})$ & $\left\langle m_{B W}\right\rangle(\mathrm{GeV})$ & $\left\langle m_{B W}^{2}\right\rangle\left(\mathrm{GeV}^{2}\right)$ & $\left\langle m_{B W}^{3}\right\rangle\left(\mathrm{GeV}^{3}\right)$ & $\left\langle m_{B W}^{4}\right\rangle\left(\mathrm{GeV}^{4}\right)$ \\
\hline 171 & $148.76(148.08)$ & $2.24(2.21) \times 10^{4}$ & $3.41(3.35) \times 10^{6}$ & $5.24(5.11) \times 10^{8}$ \\
\hline 173 & $150.44(149.56)$ & $2.29(2.26) \times 10^{4}$ & $3.53(3.46) \times 10^{6}$ & $5.48(5.32) \times 10^{8}$ \\
\hline 175 & $152.18(151.00)$ & $2.35(2.30) \times 10^{4}$ & $3.66(3.56) \times 10^{6}$ & $5.74(5.54) \times 10^{8}$ \\
\hline 177 & $153.80(152.60)$ & $2.40(2.36) \times 10^{4}$ & $3.77(3.67) \times 10^{6}$ & $5.99(5.78) \times 10^{8}$ \\
\hline 179 & $155.61(153.97)$ & $2.45(2.40) \times 10^{4}$ & $3.91(3.78) \times 10^{6}$ & $6.28(6.00) \times 10^{8}$
\end{tabular}

\subsection{Conclusions}

I investigated some contributions to the hadronization systematics on the top mass measurement, namely the impact of the bottom-quark fragmentation and non-perturbative effects in the definition of the reconstructed top mass. HERWIG and PYTHIA, the two most popular parton shower and hadronization models, were retuned to $e^{+} e^{-} \rightarrow b \bar{b}$ data from LEP and SLD, but nevertheless, even after the fit, they exhibit some relevant discrepancies. Differences are also observed when comparing with NLO calculations for quantities relying on top decays, such as the $B$-lepton invariant mass in the dilepton channel. This finding is clearly a signal that bottom fragmentation is an issue which has to be further tackled to make a statement on its contribution to the uncertainties on the top quark mass. As for the Monte Carlo codes, using the object-oriented HERWIG++ and PYTHIA 8, improving both shower and hadronization models, is mandatory in order to understand better the difference with respect to the NLO computation. Also, a direct measurements of $b$-fragmentation in top decays at the LHC will allow a direct fit of the hadronization models and therefore a test of factorization as well, after comparing with the results in $e^{+} e^{-}$annihilation.

I also discussed the relation between the measured so-called Monte Carlo mass and theoretical definitions like the pole mass. Since the top mass is reconstructed from the kinematic properties of final-state leptons and jets, using Monte Carlo programs which neglect interference effects between production and decay phases should lead to the measurement of a quantity close to the pole mass, as this is defined in the on-shell renormalization scale. In fact, calculations carried out in the framework of Soft Collinear Effective Theory have displayed that the Monte Carlo mass, determined as the jet mass at a scale given by the shower cutoff, differs from the pole mass by an amount of $O\left(\alpha_{S} \Gamma_{t}\right)$. Investigation of Monte Carlo samples with hadronized top quarks, wherein the Monte Carlo mass turns to be a meson mass, may be a useful benchmark and ultimately shed some light on this issue. This is in progress as well.

\section{References}

[1] ATLAS Collaboration, Phys. Lett. B716 (2012) 1;

CMS Collaboration, Phys. Lett. B716 (2012) 30.

[2] Gfitter Group Collaboration, Eur. Phys. J. C74 (2014) 3046.

[3] ATLAS, CDF, CMS and D0 Collaborations, arXiv:1403.4427 [hep-ex].

[4] G. Degrassi, S. Di Vita, J. Elias-Miro, J. R. Espinosa, G.F. Giudice, G. Isidori and A. Strumia, JHEP 1208 (2012) 098.

[5] A. Kharchilava, Phys. Lett. B476 (2000) 73.

[6] R. Chierici and A. Dierlamm, CMS Note 2006/058. 
[7] T. Sjostrand, S. Mrenna and P. Skands, JHEP 0605 (2006) 026.

[8] G. Corcella and V. Drollinger, Nucl. Phys. B730 (2005) 82.

[9] G. Corcella and F. Mescia, Eur. Phys. J. C65 (2010) 171; Erratum-ibid. C68 (2010) 687.

[10] G. Corcella et al, JHEP 0101 (2001) 010.

[11] S. Biswas, K. Melnikov and M. Schulze, JHEP 1008 (2010) 048.

[12] A. Czarnecki and K. Melnikov, Phys. Rev. D59 (1999) 014036.

[13] B. Mele and P. Nason, Nucl. Phys. B361 (1991) 626.

[14] G. Corcella and A.D. Mitov, Nucl. Phys. B623 (2002) 247.

[15] M. Cacciari, G. Corcella and A.D. Mitov, JHEP 0212 (2002) 015.

[16] M. Cacciari and P. Nason, Phys. Rev. Lett. 89 (2002) 122003.

[17] M. Brucherseifer, F. Caola and K. Melnikov, JHEP 04 (2013) 059.

[18] J. Gao, C.S. Li and H.X. Zhu, Phys. Rev. Lett. 110 (2013) 042001.

[19] A. Denner, S. Dittmaier, S. Kallweit and S. Pozzorini, JHEP 1210 (2012) 110.

[20] S. Catani, G. Marchesini and B.R. Webber, Nucl. Phys. B349 (1991) 635.

[21] B. Andersson, G. Gustafson, G. Ingelman and T. Sjostrand, Phys. Rept. 97 (1987) 31.

[22] B.R. Webber, Nucl. Phys. B238 (1984) 492.

[23] OPAL Collaboration, Eur. Phys.J. C29 (2003) 463.

[24] ALEPH Collaboration, Phys. Lett. B512 (2001) 30.

[25] SLD Collaboration, Phys. Rev. Lett. 84 (2000) 4300.

[26] G. Corcella, talk given at TOP LHC Working Group meeting, CERN, May 2014.

[27] ATLAS Collaboration, Eur. Phys. J. C73 (2013) 2034.

[28] G. Corcella, M.L. Mangano and M.H. Seymour, JHEP 0007 (2000) 004.

[29] G. Corcella, Int. J. Mod. Phys. A 16S1A (2001) 372.

[30] T. Sjostrand, S. Mrenna and P. Skands, Comput. Phys. Commun. 178 (2008) 852.

[31] J. Bellm et al, arXiv:1310.6877 [hep-ph].

[32] G. Corcella, F. Mescia and K. Tywoniuk, in preparation.

[33] CMS Collaboration, Eur. Phys. J. C73 (2013) 2494.

[34] A. Juste et al, Snowmass White Paper, arXiv:1310.0799 [hep-ph].

[35] S. Frixione, P. Nason and C. Oleari, JHEP 0711 (2007) 070.

[36] S. Frixione and B.R. Webber, JHEP 0206 (2002) 029.

[37] S. Frixione and A.D. Mitov, JHEP 1409 (2014) 012.

[38] A.H. Hoang and I.W. Stewart, Nucl. Phys. Proc. Suppl. 185 (2008) 220.

[39] M. Czakon, P. Fiedler and A.D. Mitov, Phys. Rev. Lett. 110 (2013) 252004.

[40] CMS Collaboration, Phys.Lett. B728 (2014) 496. 
